\title{
High resolution ISAR imaging in receiver centered region area in bistatic radar
}

\author{
Long Zhang ${ }^{1,2^{*}}$, Tao Su${ }^{1}$, Zheng $\mathrm{Liu}^{1}$ and Xuehui $\mathrm{He}^{1}$
}

\begin{abstract}
Aim at overcoming limitations from a single fixed aspect in monostatic inverse synthetic aperture radar (ISAR) system, bistatic radar system becomes a hot research topic in ISAR imaging. However, it is become more difficult to obtain a high resolution ISAR image of maneuvering target in receiver centered region area of bistatic radar for time varying bistastic angle and equivalent line of sight (LOS) aspect. In this paper, a super-resolution imaging method based on Radon transform combined with time chirp distribution search (TCDS) procedure is proposed. This method attempts to estimate the chirp rate and its changing rate corresponding to high-order phase terms in cross range. After compensating the phase error of high order, more scattering centers of target are extracted and high resolution imaging is generated by Radon-TCDS-Relax algorithm. Simulation results are provided to demonstrate the performance of the proposed method.
\end{abstract}

Keywords: ISAR imaging, Bistatic radar, Time Chirp distribution Search, Super resolution, Radon transform, Relax more the scattering centers

\section{Introduction}

Inverse synthetic Radar (ISAR) imaging becomes an important approach to achieve high resolution images of moving targets from a long distance, which are very useful for automatic target recognition and other applications. For observing target from a fixed aspect, only limited target information can be obtained by a monostatic radar even through the sophisticated ISAR imaging algorithm, and thus a high resolution ISAR image is sometimes unable produced in the case of target moving along the line of sight(LOS) [1-3]. In order to overcome these limitations in conventional monostatic radar, the bistatic radar system becomes a hot research topic [3,4].

In bistatic ISAR system, the transmitter and receiver are respectively placed in different sites [5], and thus, the spatially separated Radar system can be more reliable in many ways, e.g., more useful target information can be obtained, more safer is the radar system in battlefield, longer distanced can be operated and so on, among which the most important is the more different available knowledge of target obtained by different radar aspect.

\footnotetext{
* Correspondence: zhanglong@mail.xidian.edu.cn

${ }^{1}$ National Laboratory of Radar Signal Processing, Xidian University, Xi'an 710071, Peoples R China

${ }^{2}$ College of Electronics and Information, Xi'an Polytechnic University, Xi'an
} 710048, Peoples R China

\section{Springer}

(c) 2013 Zhang et al.; licensee Springer. This is an Open Access article distributed under the terms of the Creative Commons Attribution License (http://creativecommons.org/licenses/by/2.0), which permits unrestricted use, distribution, and reproduction in any medium, provided the original work is properly cited.
However, for the separated transmitter and Receiver, the geometry of the target and radar becomes more complex and effect of the separated transmitter and receiver is obviously. Therefore, the characteristic of the bistatic ISAR system represented by two parameters, the relationship of bistatic angle and azimuth angle of equivalent line of sight (LOS) should be studied intensively. The characteristics of bistastic radar have great impacts on the model and direction of the radial wave number respectively.

This paper is organized as follows. In Section 2, the geometry model of Bistatic ISAR is developed, the effects of ISAR echoes from two parameters, bistatic angle and the azimuth angle of equivalent line of sight (LOS) are discussed, relationship between the ISAR echoes and time variation of the bistatic angle in receiver-center region is investigated. In Section 3, effect of the variation bistatic angle on the ISAR imaging is analyzed and the range envelope and cross range processing is studied in detail. In Section 4, the algorithm of combination of Time Chirp-rate Distribution Search procedure and Relax (Radon-TCDSRelax) aiming at estimating the chirp rate and quadratic chirp rate for eliminating the high order phase terms induced by bistatic angle in two polar area is proposed. In Section 5, we provide simulation results to demonstrate the performance of the proposed method. 


\section{Bistatic ISAR signal model}

\subsection{Geometry of the bistatic ISAR}

In bistatic ISAR system, target imaging is similar to that of monostatic radar: target illuminated by the transmitter and target echoes detected and processed by the receiver. The principle of ISAR imaging in Bistatic radar is similar to the monostatic system, in which high-rang resolution is achieved by transmitting wideband pulses and cross range resolution is obtained by coherently integrating the echoes backscattered from different aspect aspect. But the propagation of echoes from target in bistatic radar is more complicated than that of the monostatic radar [1-5]. A bistatic radar system geometry is shown in Figure 1. Two-dimensional north-referenced coordinate system is used in Figure 1, which shows the coordinate system and parameters defining bistatic radar operation in the $x, y$ plane. Baseline is the coincide with the horizontal axis and the original point $\mathrm{o}$ is location on midpoint of the baseline $L$, and the location of the transmitter and receiver is $T x(-L / 2,0)$, $R x(L / 2,0)$, respectively. The unit vector of the transmitter and receiver is $\hat{\boldsymbol{R}}_{\boldsymbol{T}}=\left[\cos \theta_{T}, \sin \theta_{T}\right]^{T}$ and $\hat{\boldsymbol{R}}_{\boldsymbol{T}}=$ $\left[\cos \theta_{T}, \sin \theta_{T}\right]^{T}$, and the vector of slant range of transmitter and receiver can be written as $\boldsymbol{R}_{\boldsymbol{R}}=R_{R} \cdot \hat{\boldsymbol{R}}_{\boldsymbol{R}}$, $\boldsymbol{R}_{\boldsymbol{T}}=\boldsymbol{R}_{T} \cdot \hat{\boldsymbol{R}}_{\boldsymbol{T}}$. The sum of $\hat{\boldsymbol{R}}_{\boldsymbol{R}}$ and $\hat{\boldsymbol{R}}_{\boldsymbol{T}}$ is $\boldsymbol{R}_{\boldsymbol{e}}=\boldsymbol{R}_{\boldsymbol{R}}+\boldsymbol{R}_{\boldsymbol{T}}=$ $2 \cos \beta \cdot\left[\cos \theta_{e}, \sin \theta_{e}\right]^{\mathrm{T}}$, which is the bisects of the angle of $\hat{\boldsymbol{R}}_{\boldsymbol{R}}$ and $\hat{\boldsymbol{R}}_{T}$. The equivalent monostatic radar is $o^{\prime}$ and LOS is $\hat{\boldsymbol{R}}_{\boldsymbol{e}}$, whose angle between axis $x$ is $\theta_{\boldsymbol{e}}$ [1]. The angle $\beta=\left(\theta_{R}-\theta_{T}\right) / 2$ is called the half bistatic angle, and $\theta_{e}=\left(\theta_{R}+\theta_{T}\right) / 2$. For simplicity of analysis, the unit vector of bisector is $\hat{\boldsymbol{R}}_{\boldsymbol{e}}=\left[\cos \theta_{e}, \sin \theta_{e}\right]^{T}$ and the moving Target unit vector is $\boldsymbol{v}=v \cdot\left[\cos \theta_{v}, \sin \theta_{v}\right]^{\mathrm{T}}$. If the three of parameters $\theta_{R}, \theta_{T}, L$ and $R$ are known, the remaining can be decided by equ. (25) in literature [1], and thus the geometry model of the bistatic can be determined. The variation of $\beta$ can result in the variation in support

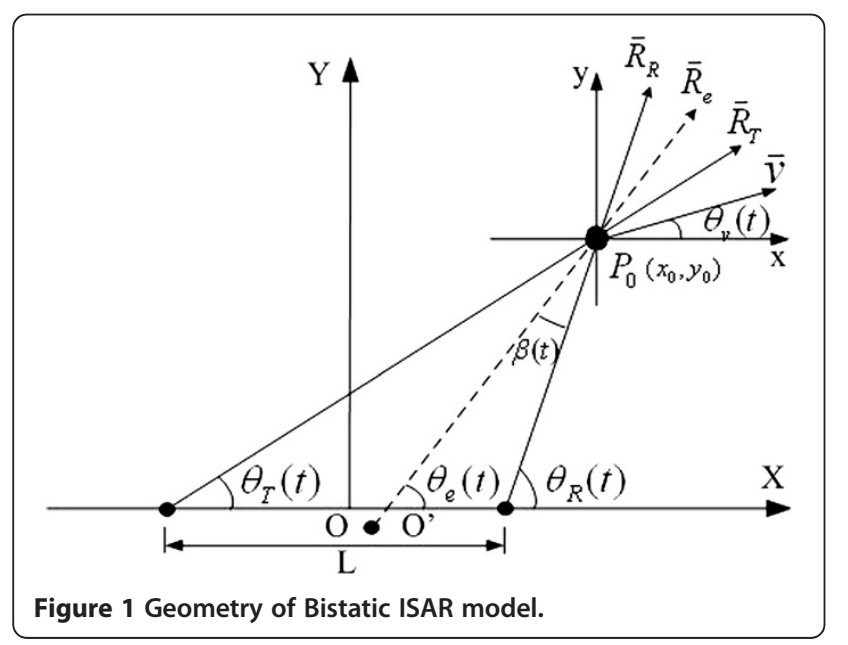

region, which has effect on the range resolution. Similarly, the varied $\theta_{e}$ of each of echoes has effect on the cross range resolution. Therefore, the parameters $\beta_{m}$ and $\theta_{\text {em }}$ are the two key parameters whose values and variations have significantly effect on the characteristic of echoes.

\subsection{Receiver centered region ISAR area}

In bistatic radar system the bistastic angle is the most crucial parameter which significantly affects the performance of imaging algorithm and the quality of ISAR image. The larger the bistastic angle, the greater effects of the separate transmitter and receiver will take on. Therefore, it is more important to study the variation of bistastic angle of target by observed radar from different operating regions observation region. These differences can be defined by bistatic angle isorange contour on the bistatic plane. Bistatic angle isorange contour is a group of circles with their center located at $(0, L \cot 2 \beta / 2)$ and radiu $r=L / 2 \sin 2 \beta$, as shown in Figure 2. The equation of circle of the isorange contour of bistatic angle as follows

$$
x^{2}+\left(y-\frac{L \cot 2 \beta}{2}\right)^{2}=\left(\frac{L}{2 \sin 2 \beta}\right)^{2}
$$

In general, for bistatic radar, four distinct operating regions can be defined: receiver-centered region, transmitter-centered region, the receiver transmitter centered region, or simply the cosite region and front scattering region. In practice, the most interest region is the area around the receiver.

In the receiver-centered region $R_{R}<<R_{T}, R_{R}<L / 2$, the system has a long operating range and a high precision, but the coverage is decreased. The bistastic angle is relatively large which may result in a worsen range resolution. With the initial condition $R_{R}<<R_{T}$, we have the equivalent

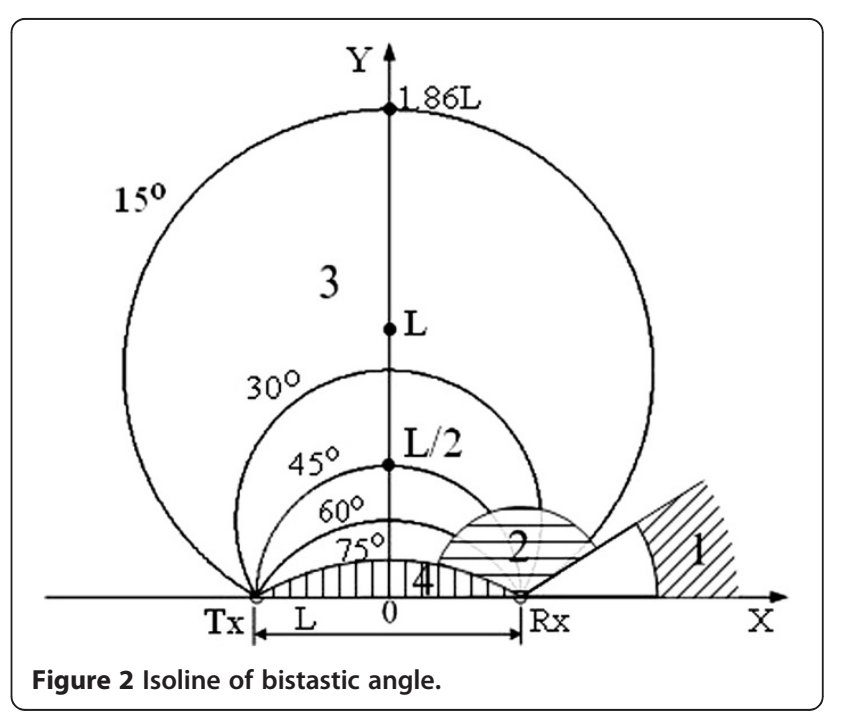


rotation angular velocity $\omega_{e 0} \approx \nu \sin \left(\theta_{R}-\theta_{v}\right) / 2 R_{R}$. If the high cross range resolution can be achieved, $\left|\theta_{R}-\theta_{v}\right| \rightarrow 90^{\circ}$ must hold, we thus have

$$
\begin{gathered}
\left|\omega_{\beta 0}\right|=\frac{v}{2 R_{R}}\left|\sin \left(\theta_{R}-\theta_{v}\right)\right| \rightarrow \frac{v}{2 R_{R}} \\
\left|\omega_{e}^{\prime}\right|=\frac{v^{2}}{2 R_{R}^{2}}\left|\sin 2\left(\theta_{R}-\theta_{v}\right)\right| \rightarrow \frac{v^{2}}{2 R_{R}^{2}}
\end{gathered}
$$

When the condition $v<<R_{R}$ is not satisfied and the variation of the bistastic angle is relatively large. As a result, accelerate angular rate is considerable and cannot be ignored. The target must be treated as undergoing irregular rotation, and therefore the range envelope of each of echoes should be aligned before employing the range instantaneous Doppler (RID) algorithm in order to achieve the high resolution ISAR image.

In this paper, we mainly focus on the ISAR image in the receiver centered region. For other operating region detection and imaging methods, the reader is referred to literature $[2,3,5]$.

\subsection{Signal model}

Assume that the transmitted wideband signal is

$$
s\left(\hat{t}, t_{m}\right)=u_{c}(\hat{t}) e^{j 2 \pi f_{c}\left(\hat{t}+t_{m}\right)}
$$

where $u_{c}(\hat{t})=A_{c} \operatorname{rect}\left(\hat{t} / T_{p}\right) e^{j \pi \mu \hat{t}^{2}}$ is the subpulse of chirp, $\hat{t}$ is the fast time, $\mu=B / T_{p}$ is the chirp rate, $\mathrm{B}$ is the bandwidth, $T_{P}$ is the pulse width, and $t_{m}=m T_{r}$ ( $m=0,1,2, \cdots, M-1)$ is slow time with $T_{r}$ being the pulse repetition period.

Let $\vec{v}=v \cdot\left[\cos \theta_{v}, \sin \theta_{v}\right]^{T}$ denote velocity vector, and $R_{T}\left(t_{m}\right)$ and $R_{R}\left(t_{m}\right)$ are the distances between the two radars (Radar A and Radar B) and a focusing point $o$ on the target, $\theta_{T}\left(t_{m}\right), \theta_{R}\left(t_{m}\right)$ denote the aspect angle of target to the transmitter and receiver at time $t_{m}$, respectively. Therefore $\theta_{e}\left(t_{m}\right)=\left[\theta_{R}\left(t_{m}\right)+\theta_{T}\left(t_{m}\right)\right] / 2$ and $\beta\left(t_{m}\right)=$ $\left[\theta_{R}\left(t_{m}\right)-\theta_{T}\left(t_{m}\right)\right] / 2$ denote the composite angle and half bistatic angle are at time $t_{m}$. At time $t_{m}=0$, we have the range of scatter $P_{n}$ on target to radar

$$
\begin{aligned}
R_{n T}(0)= & {\left[\left(R_{T 0} \cos \theta_{T 0}+x_{n} \cos \theta_{e 0}-y_{n} \sin \theta_{e 0}\right)^{2}\right.} \\
& \left.+\left(R_{T 0} \sin \theta_{T 0}+x_{n} \sin \theta_{e 0}+y_{n} \cos \theta_{e 0}\right)^{2}\right]^{1 / 2} \\
& \approx R_{T 0}+x_{n} \cos \left(\theta_{e 0}-\theta_{T 0}\right)-y_{n} \sin \left(\theta_{e 0}-\theta_{T 0}\right)
\end{aligned}
$$

$$
\begin{aligned}
R_{n R}(0)= & {\left[\left(R_{R 0} \cos \theta_{R 0}+x_{n} \cos \theta_{e 0}-y_{n} \sin \theta_{e 0}\right)^{2}\right.} \\
& \left.+\left(R_{R 0} \sin \theta_{R 0}+x_{n} \sin \theta_{e 0}+y_{n} \cos \theta_{e 0}\right)^{2}\right]^{1 / 2} \\
& \approx R_{R 0}+x_{n} \cos \left(\theta_{e 0}-\theta_{R 0}\right)-y_{n} \sin \left(\theta_{e 0}-\theta_{R 0}\right)
\end{aligned}
$$

where $\theta_{e 0}, \theta_{T 0}, \theta_{R 0}, R_{T 0}$ and $R_{R 0}$ are values corresponding to $\theta_{e}, \theta_{T} \theta_{R}, R_{T}$ and $R_{R}$, respectively. At initial time $t_{0}$, we can obtain the equivalent slant range

$$
\begin{aligned}
R_{n}\left(t_{m}\right) & =\left[R_{n T}\left(t_{m}\right)+R_{n R}\left(t_{m}\right)\right] / 2 \\
& =R_{V}\left(t_{m}\right)+R_{n \Omega}\left(t_{m}\right)
\end{aligned}
$$

$$
R_{V}\left(t_{m}\right)=\left[R_{T}\left(t_{m}\right)+R_{R}\left(t_{m}\right)\right] / 2
$$

$$
R_{n \Omega}\left(t_{m}\right)=\left\{x_{n} \cos \left[\theta_{e 0}-\theta_{e}\left(t_{m}\right)\right]-y_{n} \sin \left[\theta_{e 0}-\theta_{e}\left(t_{m}\right)\right]\right\} \cdot \cos \beta\left(t_{m}\right)
$$

In (7), $R_{V}\left(t_{m}\right)$ denote the translational motion and performing the Taylor series expansion up to the second order term yields

$$
R_{V}\left(t_{m}\right) \approx R_{0}+V \cdot t_{m}+\frac{1}{2} \dot{V} \cdot t_{m}^{2}
$$

where $R_{0}=\left[R_{R}(0)+R_{T}(0)\right] / 2$ denotes the initial slant range , $V$ and ' $V$ denote the first and second derivatives of translational motion with respect to $t$, respectively, $R_{n \Omega}\left(t_{m}\right)$ denotes the rotational motion about the $o^{\prime}$, when $v<<R_{R}$ does not hold, the variation of bistastic angle is relatively great and the acceleration of $\theta_{e}$ cannot be neglected, which target undergoing irregular rotation. Using the Taylor expansion in second-order series of $\beta\left(t_{m}\right)$ and $\theta_{e}\left(t_{m}\right)$, we can obtain the simplified approximation of $A n(t)$ as follows:

$$
\beta\left(t_{m}\right) \approx \beta_{0}+\Omega_{\beta} \cdot t_{m}+\frac{1}{2} \dot{\Omega}_{\beta} \cdot t_{m}^{2}
$$

$$
\theta_{e}\left(t_{m}\right)=\theta_{e 0}+\Omega_{e} \cdot t_{m}+\frac{1}{2} \dot{\Omega}_{e} \cdot t_{m}^{2}
$$


After substituting (11) and (12) in (9), we can have

$$
\begin{aligned}
R_{n \Omega}\left(t_{m}\right) \approx\{ & x_{n} \cos \left[\theta_{e 0}-\theta_{e}\left(t_{m}\right)\right] \\
& \left.-y_{n} \cos \left[\theta_{e 0}-\theta_{e}\left(t_{m}\right)\right]\right\} \cdot \cos \left(\beta_{e}\left(t_{m}\right)\right) \\
= & x_{n} \cos \beta_{0}-\left(y_{n} \cos \beta_{0} \Omega_{e}+x_{n} \sin \beta_{0} \Omega_{\beta}\right) t_{m} \\
& +\left\{\left[\frac{1}{2} x_{n}\left(\Omega_{e}^{2}+\Omega_{\beta}^{2}\right)-y_{n} \dot{\Omega}_{e}\right] \cdot \cos \beta_{0}\right. \\
& \left.+\left(y_{n} \Omega_{e} \Omega_{\beta}-\frac{1}{2} x_{n} \dot{\Omega}_{\beta}\right) \cdot \sin \beta_{0}\right\} t_{m}^{2} \\
& -\left[\frac{1}{2} y_{n} \cos \beta_{0} \Omega_{e} \Omega_{\beta}^{2}+\frac{1}{2}\left(x_{n} \Omega_{e}^{2} \Omega_{\beta}\right.\right. \\
& \left.\left.-y_{n} \dot{\Omega}_{e} \Omega_{\beta}+y_{n} \Omega_{e} \dot{\Omega}_{\beta}\right) \sin \beta_{0}\right] t_{m}^{3}+O\left(t_{m}^{3}\right)
\end{aligned}
$$

where $O\left(t_{m}^{3}\right)$ denotes the terms higher than third order and can be ignored. The baseband echo signal can be written as [6]

$$
s\left(\hat{t}, t_{m}\right)=\sum_{n} A_{n} \cdot u_{c}\left(\hat{t}-\frac{2 R_{n}\left(t_{m}\right)}{c}\right) \cdot \exp \left[-j \frac{4 \pi}{c} f_{c} R_{n}\left(t_{m}\right)\right]
$$

where $A_{n}$ denote the amplitude of scatterers. After range-matched filtering, (14) becomes

$$
\begin{aligned}
s\left(\hat{t}, t_{m}\right)= & \sum_{n} A_{n} \cdot \operatorname{Sinc}\left[B\left(\hat{t}-\frac{2 R_{n}\left(t_{m}\right)}{c}\right)\right] \\
& \times \exp \left[-j \frac{4 \pi}{c} f_{c} R_{n}\left(t_{m}\right)\right]
\end{aligned}
$$

In bistatic ISAR system, the bistastic angle in (13) is no longer to be ignored and its variation depends on the velocity and aspect angle relative to LOS. Variation of bistatic angle has an influence on the rotational motion and has no effect on translational motion. Therefore, the translational motion compensation method is similar to the monostatic ISAR system.

\section{Effect on the envelope and phase from bistatic angle in receiver centered region}

When the medium resolution involved, the variation of bistatic angle can be ignored for uniformed motion target, therefore we have $\Omega_{\beta}=0$ and get $R_{n \Omega}\left(t_{m}\right)=\left(x_{n}-\right.$ $\left.y_{n} \Omega_{e} \cdot t_{m}\right) \cdot \cos \beta_{0}$, in which case an FFT-based approach may be sufficient to generate the ISAR image of target. While when high resolution images are required it is necessary to study the effect of the ISAR image when bistatics angle $\Omega_{\beta} \neq 0$.

The faster motion target moving and shorter distance of target to receiver radar, the more complexity in bistatic ISAR imaging become. Typically, migration resolution cell phenomenon is the prominent problem to be concerned. Because of the great variation of bistaics angle $\beta$ and equivalent LOS $\theta_{\text {em }}$, therefore MTRC phenomenon cannot be ignored. After compensating for the translational motion, the signal in the spatial frequency domain can be expressed as

$$
s\left(f, t_{m}\right)=\sum_{n} A_{n} \exp \left[-j \frac{4 \pi}{c}\left(f_{c}+f_{r}\right) R_{n \Omega}\left(t_{m}\right)\right]
$$

where $f \in[-B / 2, B / 2]$. Ignoring the term higher than the third order, we have

$$
R_{n \Omega}\left(t_{m}\right)=R_{\Omega}+R_{\Omega}^{\prime} t_{m}+R_{\Omega}^{\prime \prime} t_{m}^{2}+R_{\Omega}^{\prime \prime \prime} t_{m}^{3}
$$

where

$$
\begin{aligned}
R_{\Omega}= & x_{n} \cos \beta_{0} \\
R_{\Omega}^{\prime}= & -\left(y_{n} \cos \beta_{0} \Omega_{e}+x_{n} \sin \beta_{0} \Omega_{\beta}\right) \\
R_{\Omega}^{\prime \prime}= & \left\{\left[\frac{1}{2} x_{n}\left(\Omega_{e}^{2}+\Omega_{\beta}^{2}\right)-y_{n} \dot{\Omega}_{e}\right] \cdot \cos \beta_{0}\right. \\
& \left.+\left(y_{n} \Omega_{e} \Omega_{\beta}-\frac{1}{2} x_{n} \dot{\Omega}_{\beta}\right) \cdot \sin \beta_{0}\right\} \\
R_{\Omega}^{\prime \prime \prime}= & -\left[\frac{1}{2} y_{n} \cos \beta_{0} \Omega_{e} \Omega_{\beta}^{2}+\frac{1}{2}\left(x_{n} \Omega_{e}^{2} \Omega_{\beta}\right.\right. \\
& \left.\left.-y_{n} \dot{\Omega}_{e} \Omega_{\beta}+y_{n} \Omega_{e} \dot{\Omega}_{\beta}\right) \sin \beta_{0}\right]
\end{aligned}
$$

Substituting (17) into (16), we have

$$
\begin{aligned}
s\left(f, t_{m}\right)= & \sum_{n} A_{n} \exp \left[-j \frac{4 \pi}{c}\left(f_{c}+f_{r}\right)\right. \\
& \left.\left(R_{\Omega}+R_{\Omega}^{\prime} t_{m}+R_{\Omega}^{\prime \prime} t_{m}^{2}+R_{\Omega}^{\prime \prime \prime} t_{m}^{3}\right)\right]
\end{aligned}
$$

The remainder of this section is evaluating the variation the bistatic angle and composite LOS angle in receiver centered region. Bistatic sytem operation at $\mathrm{L}$ band and bandwidth is $400 \mathrm{MHz}$, baseline is $100 \mathrm{Km}$. The range of target to receiver radar is $6 \mathrm{~km}$, and target moving along the $\mathrm{x}$-axis and its velocity is $\mathrm{v}=400 \mathrm{~m} / \mathrm{s}$. The variation value of equivalent monostatic view angle is shown as Figure 3a, The total varation $\Delta \theta=-10.97^{\circ}$, and variation value of bistastic angle is shown in Figure 3b. $\Delta \beta=-10.84^{\circ}$. Variation rate of $\beta$ and $\theta_{e}$ are shown in Figure $3 \mathrm{c}$ and Figure $3 \mathrm{~d}$, respectively. As we 
(a)

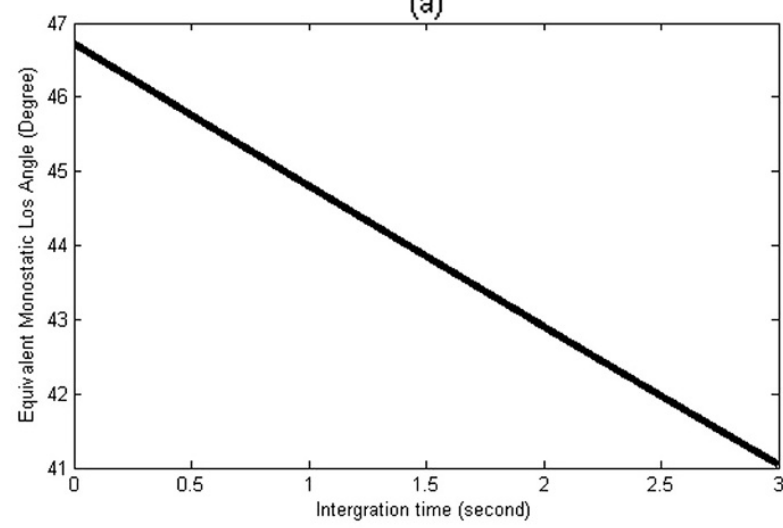

(c)

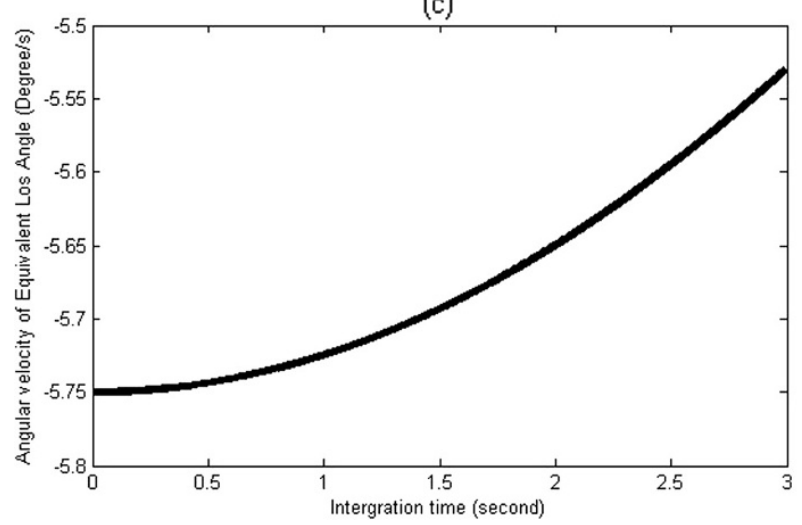

(b)

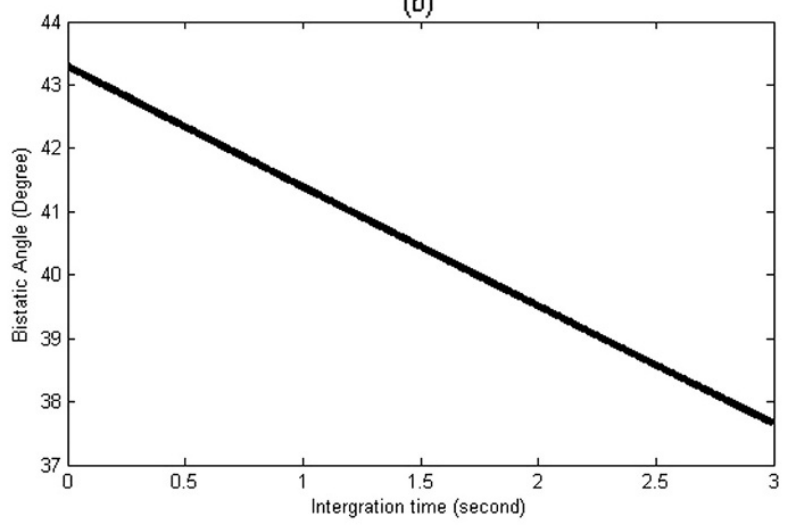

(d)

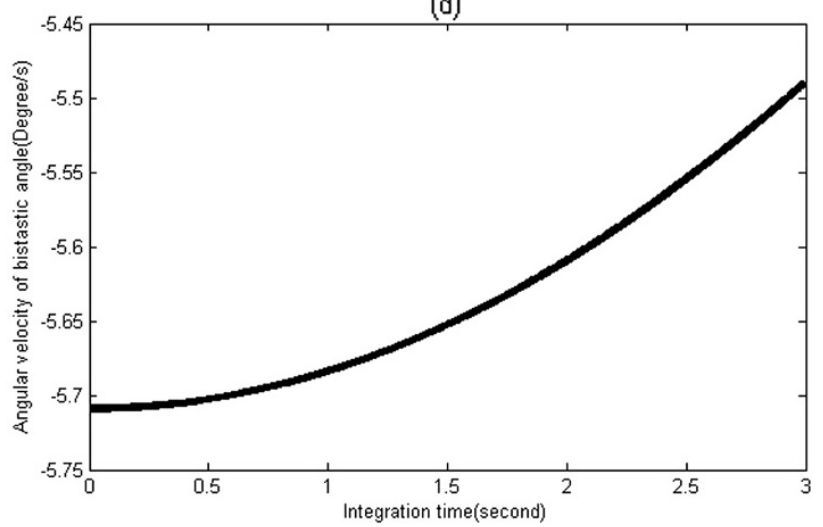

Figure 3 bistastic/ Equivalent Monostatic angle variation: (a) Variation of Equivalent Monostatic angle. (b) Variation of bistastic angle. (c) Variation rate of Equivalent Monostatic angle. (d) Variation rate of bistastic angle.

can see, although coherent integration time is 3 seconds, the angular velocity of $\beta$ and $\theta_{e}$ are present nonlinear characteristic. The minimum value arrive at the $0.099 \mathrm{rad} / \mathrm{s}$ and $0.098 \mathrm{rad} / \mathrm{s}$. Therefore, the MTRC phenomenon cannot be neglected.

In following we will discuss the scatterers migration througth resolution cells conditions

(1) Migration range cell from the bistastic angle. Let $D_{r}$ and $D_{a}$ are the maximum range and cross range dimensions of target, respectively. Because of the $\theta_{e}$ variation, $\sin \theta_{e}$ series are approximated by first order, the condition can be written as [7] (isorange contour of bistatic angle)

$$
D_{r}<\frac{32 \rho_{a}^{2} \rho_{r}}{\lambda^{2}}
$$

From the literature [7] we know that, the closer of range between target to transmitter/receiver, the denser isorange contour of bistatic angle are presented the greater variation rate is. Therefore, when higher resolution demanded and greater size as high velocity of the target involved, the more obvious effect from bistastic will take on. When the (23) does not satisfied, migration range cell will happens.

(2) Migration in cross range cell, for the variation $\theta_{e}$, the $\cos \theta_{e}$ series are approximated by second order, the condition can be written as

$$
D_{a}<\frac{24 \rho_{a}^{3}}{\lambda^{2}}
$$

When bandwidth $\mathrm{B}=400 \mathrm{MHz}$,centroid frequency $\mathrm{fc}=1.0 \mathrm{GHz}$, and rotational veloceity $\mathrm{w}=0.09 \mathrm{rad} / \mathrm{s}$, from (23) and (24) we can derive $D_{r}<37.56 \mathrm{~m}$ and $D_{a}$ $<39.87 \mathrm{~m}$, respectively. We can see that when the small size plane target involved and the conditions of (24) cannot be satisfied, the MTRC effect from third-order phase term of $\theta_{e}$ which could not be eliminate by RID algorithm must be considered.

\section{Super resolution algorithm in receiver centered region} In this section, we will propose new algorithm aim at estimating instantaneous Doppler, chirp rate and quadratic 
chirp rate spectrum of signal components which suitable for generating high resolution ISAR image in receiver centered region. The algorithm use randon transforming TCD of echoes and employing the Relax searching iterative procedure which has excellent performance of global optimum parameter estimating in super resolution ISAR imaging.

TCD was studied and described by O'Neill [8-10]. It is suitable for the estimation of the instantaneous chirp rate spectrum. A quadratic frequency modulated signal has the following form

$$
\begin{aligned}
s(t) & =\exp [j \Phi(t)] \\
& =\exp \left[j\left(\Phi_{0}+f \cdot t+\frac{1}{2} \mu \cdot t^{2}+\frac{1}{6} \dot{\mu} \cdot t^{3}\right)\right]
\end{aligned}
$$

where $\Phi_{0}$ is the initial phase, and $f$ is the initial frequency, $\mu$ is defined as the chirp rate and $\dot{\mu}$ is the change rate of chirp, called the quadratic chirp rate. The timechirp distribution of (25) can be written as

$$
\begin{aligned}
T C D_{s}(t, c) & =\int Z_{s}(t, \tau) \exp (-j c \tau) d \tau \\
& =\int \exp \{-j \tau[c-\dot{\mu}(t)]\} d \tau \\
& =2 \pi \delta[c-\dot{\mu}(t)]
\end{aligned}
$$

$$
Z_{s}(t, \tau)= \begin{cases}s(t+\sqrt{\tau}) s^{*}(t) s^{*}(t) s(t-\sqrt{\tau}), & \tau \geq 0 \\ Z_{s}^{*}(t,-\tau), & \tau<0\end{cases}
$$

where $\mu(t)=\mu+\dot{\mu} \cdot t$ denotes instantaneous chirp rate, and $\delta(\cdot)$ is the delta function. Apparently, the TCD of quadratic frequency modulated (QFM) signal is localized along the linear instantaneous chirp rate $\mu(t)$. Take Radon transform of signal on TCD plane and peak location can be used to estimate the chirp rate $\mu$ and quadratic chirp rate $\dot{\mu}$. The interfering cross-terms also presents in the TCD plane when multiple signals exist at the same time. Therefore the energy of signal should be integrated in the same way as used in the Radon-Wigner transform [11] to eliminate the interference of crossterms in practice. To illustrate the TCD of QFM signal, a single component QFM and its Radon transform result is shown in Figure 4.

Based on the analysis above, the signal of target in receiver centered region is the QFM forms, so we propose a novel algorithm suit for imaging maneuvering target in the receiver centered region. That is Radon time chirp distribution Relax algorithm.

After translation motion compensation including range cell alignment and phase autofocus, we proposed the techniques focus on general cross range phase parameter estimation. We name the algorithm the Radon-TCDS-Relax.

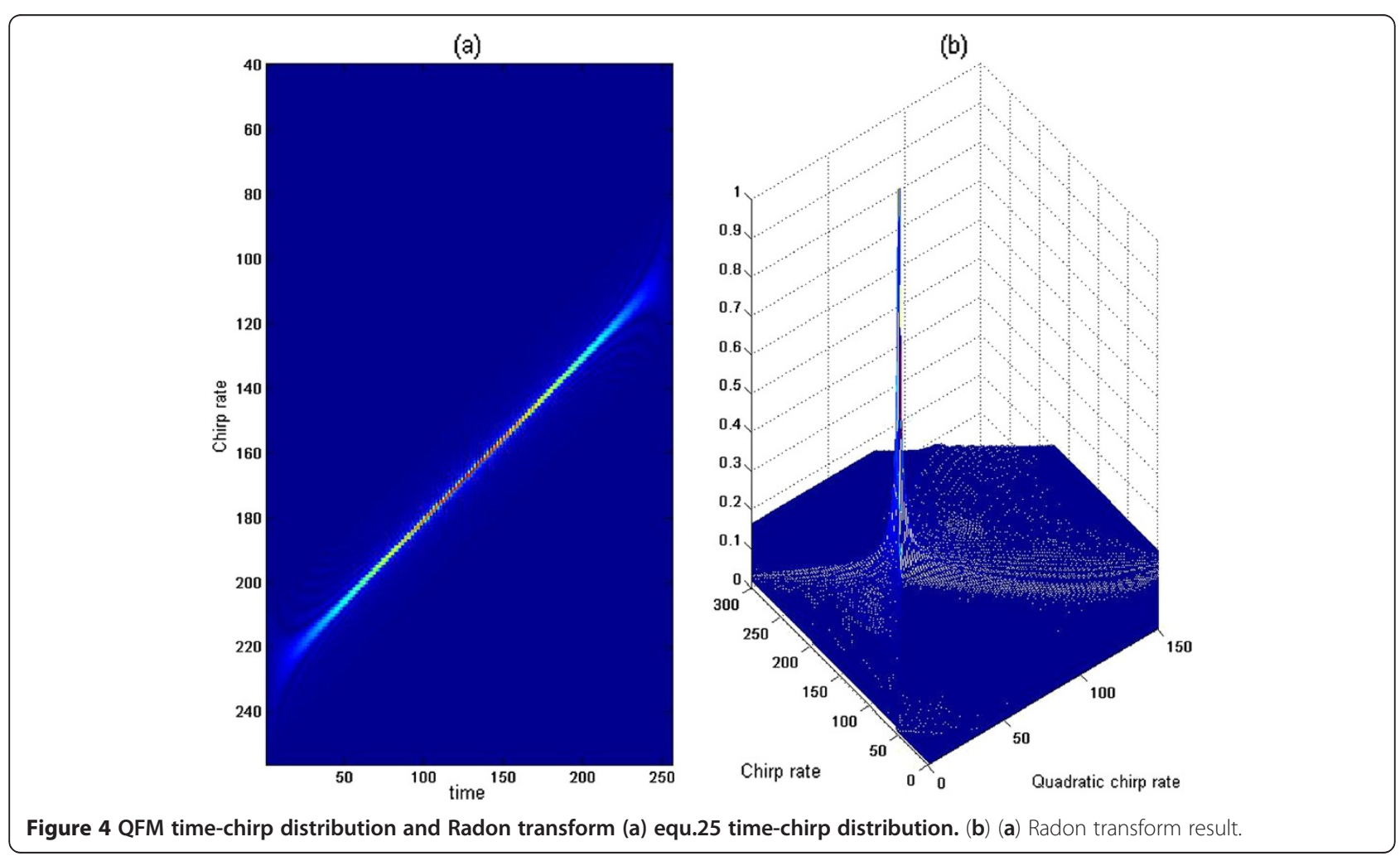


For an arbitrary range cell, the signal of target can be modeled as a sequence $s(n)$ that consists of $K$ complex QFM signal in the presence of AR noise [12-14]

$$
s(n)=\sum_{k=1}^{K} \alpha_{k} \exp \left(j 2 \pi f_{k} n+j \pi \mu_{k} n^{2}+\frac{1}{3} j \pi \dot{\mu}_{k} n^{3}\right)+e(n)
$$

where $n=0,1, \cdots N-1, N$ is the sample number in slow time, $e(n)$ is AR noise.

As we know, the parametric relaxation based algorithm suitable for estimation the complex amplitude and sinusoidal frequency. For expanding Relax to chirp signal model, let

$$
\mathbf{y}=\left[\begin{array}{llll}
y(0) & y(1) & \cdots & y(N-1)
\end{array}\right]^{T}
$$

and

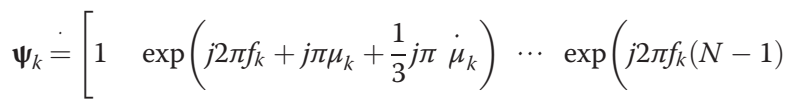

$$
\begin{aligned}
& \left.\left.+j \pi \mu_{k}(N-1)^{2}\right)+\frac{1}{3} j \pi \dot{\mu}_{k}(N-1)^{3}\right]^{T}
\end{aligned}
$$

where $[\cdot]^{T}$.

The parameters $\left\{\alpha_{k}, f_{k}, \mu_{k}, \dot{\mu}_{k}\right\}(k=1,2 \cdots K)$ can be estimation via minimizing the follwong nolinear least squares (NLS) criterion:

$$
C_{1}\left(\left\{\alpha_{k}, f_{k}, \mu_{k}, \dot{\mu}_{k}\right\}_{k=1}^{K}\right)=\left\|\mathbf{y}-\sum_{k=1}^{K} \alpha_{k} \boldsymbol{\Psi}_{k}\right\|^{2}
$$

where $\|\cdot\|$ denote Euelidean norm.

The minimization of the cost function $C_{1}$ in equ.32 is a complicated NLS optimization problem. Based on the extension of relaxation in [14], the simple algoritm for minimizing $C_{1}$ are presented. We have the following

$$
\mathbf{y}_{k}=\mathbf{y}-\sum_{i=1, i \neq k}^{K} \hat{\alpha}_{i} \hat{\psi}_{i}
$$

Assume that the parameters $\left\{\hat{\alpha}_{i}, \hat{f}_{i}, \hat{\mu}_{i}, \hat{\dot{\mu}}_{k}\right\}(i=1$, $2 \cdots K, i \neq k)$ have been estimated. Minimizing (32) is equivalent to Minimizing the following cost function

$$
C_{2}\left(\alpha_{k}, f_{k}, \mu_{k}, \dot{\mu}_{k}\right)=\left\|\mathbf{y}_{k}-\alpha_{k} \boldsymbol{\Psi}_{k}\right\|^{2}
$$

The minimization of $C_{2}$ with respect to $\alpha_{k}$ to obtain the estimate of $\alpha_{k}$

$$
\hat{\alpha}_{k}=\left(\boldsymbol{\psi}_{k}^{H} \boldsymbol{\Psi}_{k}\right)^{-1} \boldsymbol{\psi}_{k}^{H} \mathbf{y}_{k}=\boldsymbol{\psi}_{k}^{H} \mathbf{y}_{k} / N
$$

where $(.)^{H}$ denotes the complex conjugate transpose and

Letting

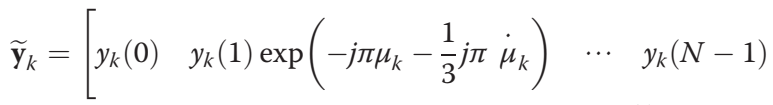

$$
\begin{aligned}
& \left.\exp \left(-j \pi \mu_{k}(N-1)^{2}-\frac{1}{3} j \pi \cdot \mu_{k}(N-1)^{3}\right)\right]^{T} \\
& \widetilde{\psi}_{k}=\left[\begin{array}{llll}
1 & \exp \left(j 2 \pi f_{k}\right) & \cdots & \exp \left(j 2 \pi f_{k}(N-1)\right)
\end{array}\right]^{T}
\end{aligned}
$$

Then we can have

$$
\hat{\alpha}_{k}=\widetilde{\psi}_{k}^{H} \widetilde{\mathbf{y}}_{k} / N
$$

From (39), we note that $\hat{\alpha}_{k}$ is the normalization of discrete Fourier transform of $\widetilde{\mathbf{y}}_{k}$, and thus $\hat{\alpha}_{k}$ can be computed by using the FFT. Assume $\mathbf{y}_{k}$ only comprise the monocomponent chirp signal, thus $\widetilde{\mathbf{y}}_{k}$ is the time unvaried signal after dechirped, therefore, the parameter can be obtained by using FFT.

After substituting the estimated $\hat{\alpha}_{k}$ into (35), the estimation value $\hat{f}_{k}, \hat{\mu}_{k}, \hat{\mu}_{k}$ of $f_{k}, \mu_{k}, \mu_{k}$ can be optimized the following

$$
\begin{aligned}
C_{3}\left(f_{k}, \mu_{k}, \dot{\mu}_{k}\right) & =\left\|\left(\mathbf{I}-\boldsymbol{\Psi}_{k} \boldsymbol{\Psi}_{k}^{H} / N\right) \mathbf{y}_{k}\right\|^{2} \\
& =\mathbf{y}_{k}^{H} \mathbf{y}_{k}-\mathbf{y}_{k}^{H} \boldsymbol{\Psi}_{k} \boldsymbol{\Psi}_{k}^{H} \mathbf{y}_{k} / N
\end{aligned}
$$

where I denotes the identity matrix.

Minimizing $C_{3}$ in equ. 38 is equivalent to maximizing

$$
C_{4}\left(f_{k}, \mu_{k}, \dot{\mu}_{k}\right)=\left(\boldsymbol{\Psi}_{k}^{H} \mathbf{y}_{k}\right)^{H} \boldsymbol{\Psi}_{k}^{H} \mathbf{y}_{k} / N
$$


So, the estimation of $f_{k}, \mu_{k}, \dot{\mu}_{k}$ can be determined by

$$
\left\{\hat{f}_{k}, \hat{\mu}_{k}, \hat{\dot{\mu}}_{k}\right\}=\arg \max _{f_{k}, \mu_{k}}\left\{\left|\widetilde{\psi}_{k}^{H} \widetilde{\mathbf{y}}_{k}\right|^{2} / N\right\}
$$

For a fixed $\mu_{k}$, the cooresponding frequency value $f_{k}$ with maximizes the function in equ. 41 can be obtained by locating the dominant peak of th normalized periodogram $\left|\widetilde{\psi}_{k}^{H} \widetilde{\mathbf{y}}_{k}\right|^{2} / N$. The the estimation problem of equ. 39 equivalent to finding a $\left(\hat{\mu}_{k}, \hat{\dot{\mu}}_{k}\right)$ such that the dominant peak of $\left|\widetilde{\psi}_{k}^{H} \widetilde{\mathbf{y}}_{k}\right|^{2} / N$ is maximum over a set of possible value of $\mu_{k}$. Where $\widetilde{\mathbf{y}}_{k}$ is the function of $\left(\mu_{k}, \dot{\mu}_{k}\right)$. Therefore, it can be search the estimation value $\hat{\mu}_{k}, \hat{\dot{\mu}}_{k}$ by optimization procedure and $\hat{f}_{k}$ can be determined with equ. (40).

In the serarching procedure, the $\hat{\mu}_{k}$ and $\hat{\dot{\mu}}_{k}$ of $k$ th signal compent can be estimated by the peak of $T C D_{s}(t, c)$ obtained by Radon transforming the signal on plane. The

coordinates of peak location is the $\hat{\mu}_{k}, \hat{\dot{\mu}}_{k}$.

The steps of Radon-TCD-Relax algorithm can be summarized as follows:

Step 1. Assume $K=1$, and obtain $\left\{\hat{f}_{1}, \hat{\mu}_{1}, \hat{\dot{\mu}}_{k}, \hat{\alpha}_{1}\right\}$ by (42) and (39).

Step 2. Assume $K=2$, and compute $\mathrm{y}_{2}$ with (34) using the $\left\{\hat{f}_{1}, \hat{\mu}_{1}, \hat{\mu}_{k}, \hat{\alpha}_{1}\right\}$ obtained in step1. Obtain the $\left\{\hat{f}_{2}, \hat{\mu}_{2}, \hat{\dot{\mu}}_{2}, \hat{\alpha}_{2}\right\}$ using the (42) and (39). Next, compute $\mathrm{y}_{1}$ with (34) by using estimated $\left\{\hat{f}_{2}, \hat{\mu}_{2}, \hat{\dot{\mu}}_{2}, \hat{\alpha}_{2}\right\}$, then redetermine $\left\{\hat{f}_{1}, \hat{\mu}_{1}, \hat{\mu}_{k}, \hat{\alpha}_{1}\right\}$ from $y_{1}$. Repeat the previous two substeps until convergence is achieved.

Step 3. Assume $K=3$, and compute $\mathrm{y}_{3}$ with (34) using the $\left\{\hat{f}_{1}, \hat{\mu}_{1}, \hat{\dot{\mu}}_{k}, \hat{\alpha}_{1}\right\}$ and $\left\{\hat{f}_{2}, \hat{\mu}_{2}, \hat{\dot{\mu}}_{2}, \hat{\alpha}_{2}\right\}$ obtained in step2. Obtain the $\left\{\hat{f}_{3}, \hat{\mu}_{3}, \hat{\dot{\mu}}_{3}, \hat{\alpha}_{3}\right\}$ using the (42) and (39). Next, compute $y_{1}$ with (34) by substitute the estimated $\left\{\hat{f}_{2}, \hat{\mu}_{2}, \hat{\dot{\mu}}_{2}, \hat{\alpha}_{2}\right\}$ and $\left\{\hat{f}_{3}, \hat{\mu}_{3}, \hat{\dot{\mu}}_{3}, \hat{\alpha}_{3}\right\}$ into
(34), then redetermine $\left\{\hat{f}_{1}, \hat{\mu}_{1}, \hat{\dot{\mu}}_{k}, \hat{\alpha}_{1}\right\}$ from $y_{1}$ and (42) and (39). Next, compute $y_{2}$ by substitute the $\left\{\hat{f}_{1}, \hat{\mu}_{1}, \hat{\dot{\mu}}_{k}, \hat{\alpha}_{1}\right\}$ 和 $\left\{\hat{f}_{3}, \hat{\mu}_{3}, \hat{\dot{\mu}}_{3}, \hat{\alpha}_{3}\right\}$ into (34). Then redetermine $\left\{\hat{f}_{2}, \hat{\mu}_{2}, \hat{\dot{\mu}}_{2}, \hat{\alpha}_{2}\right\}$ by using $\mathrm{y}_{2}$ and (42) and (39). Repeat the previous two substeps until convergence is achieved.

Step 4. Remaining steps: Continue similarly until $K$ is equal to the desired or estimated number of chirp signals. In practical, the convergence can be select under a fixed small value, such as $\varepsilon=10^{-4}$ and the iterative process should be terminated.

The parameter estimation procedure is carried out for an arbitrary range cell. For the wideband radar, the scatterers on target may occupy dozens of range cells. Therefore, Radon-TCD-Searching need to be carried out in each cell and ISAR image can be obtained ultimately.

\section{Simulation result}

The transmitter and receiver in bistastic radar sytem is shown in Figure 5. The length of baseline is $L=100 \mathrm{Km}$ and distance of target to transmitter and receiver is

$R_{T}=100 \mathrm{Km}, R_{R}=6 \mathrm{Km}$,respectively. The latter of this section is to compute the variation the bistatic angle and composite LOS angle in receiver centered region. Radar operation at $\mathrm{L}$ band, and bandwidth is $B=400 \mathrm{MHz}$, length of baseline $L=100 \mathrm{Km}$. The range of target to receiver radar is $6 \mathrm{~km}$, and target moving along the $\mathrm{x}$-axis and its velocity is $\mathrm{v}=400 \mathrm{~m} / \mathrm{s}$. The bandwidth of transmitted signal $\mathrm{B}=400 \mathrm{MHz}$, and the carrier frequency $f_{0}=1200 \mathrm{MHz}$ and a L-band radar works on L-band. The initial range of transmitter and receiver are $R_{t}=100 \mathrm{Km}$, $R_{R}=6 \mathrm{Km}$. The transmitter and receiver LOS angle are $\theta_{R}=90^{\circ}, \theta_{T}=3.43^{\circ}$. The equivalent monostastic LOS angle $\theta_{e}=46.78^{\circ}$. The initial bistastic angle $\beta=43.28^{\circ}$ and the wavelength $\lambda=0.03 m$, the the coherent integration time is 3 seconds.

The simulated plane is consists of 330 point scatterers. The plane model is shown in Figure 6a, the size of plane

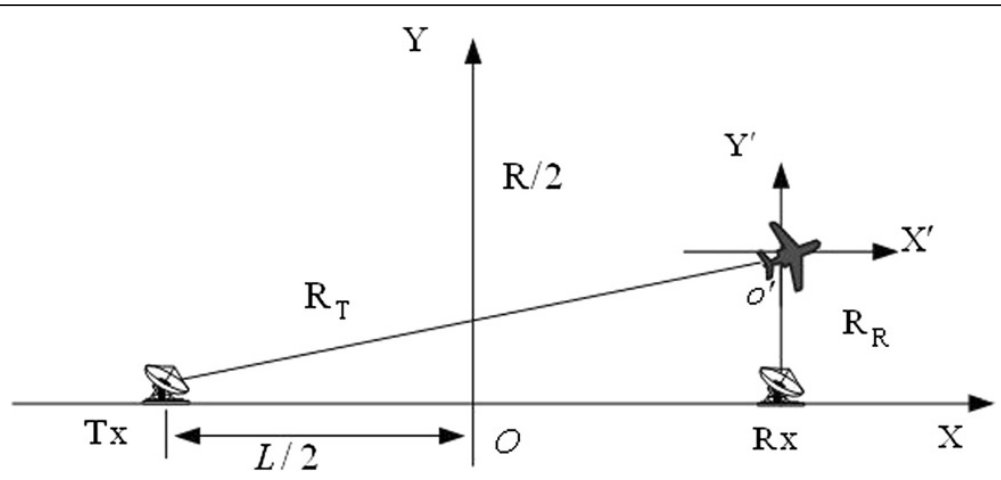

Figure 5 The plane and Bistastic model geometry. 


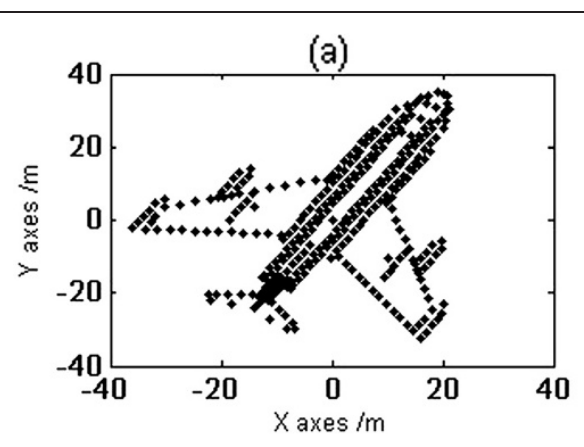

(c)

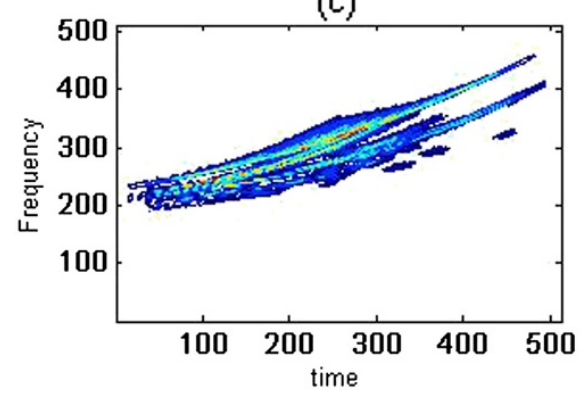

(e)

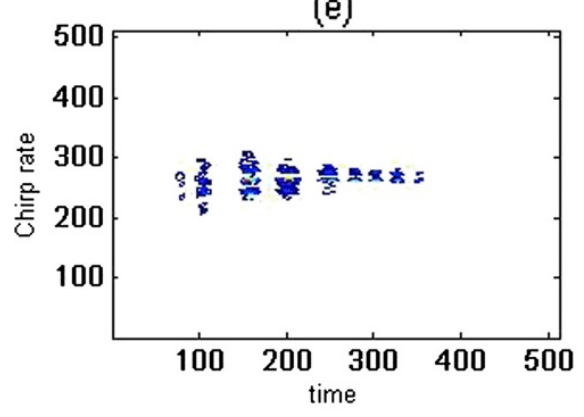

(g)

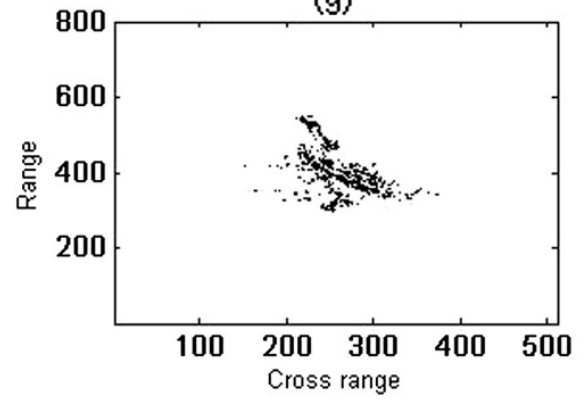

(b)

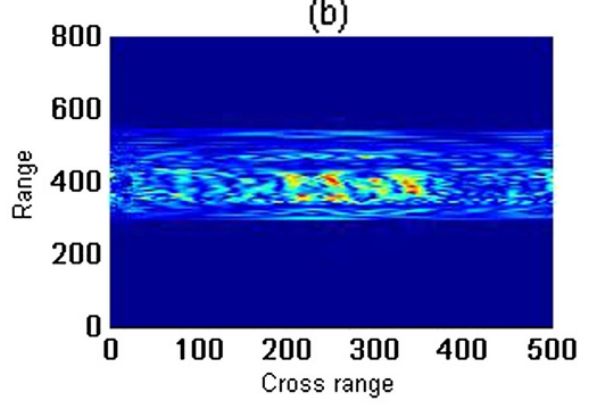

(d)

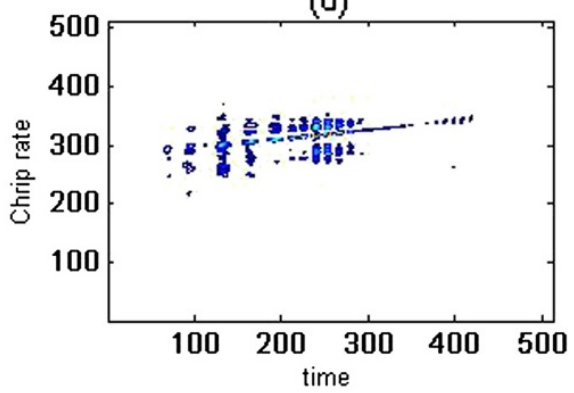

(f)

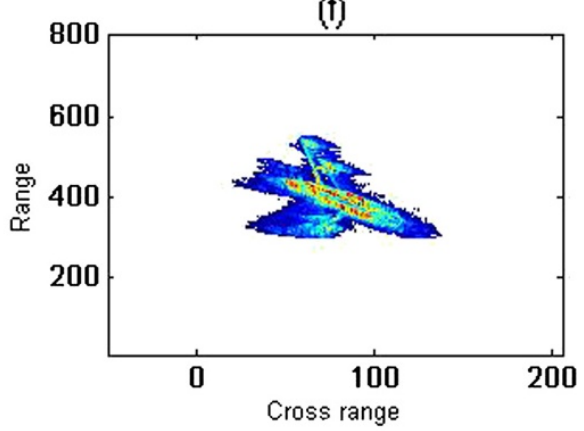

(h)

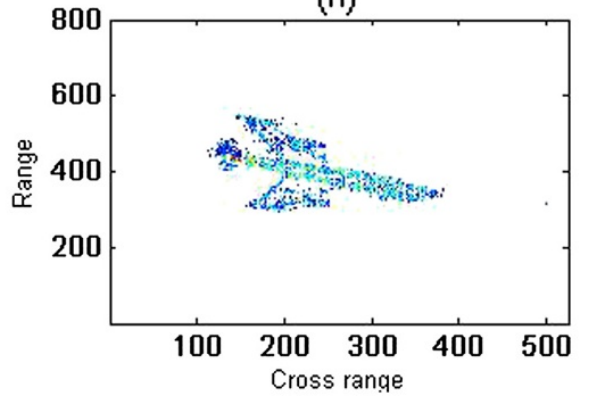

Figure 6 Simulation results (a) Yaker $\mathbf{4 2}$ model. (b) envelope after alignment. (c) time frequency distribution. (d) t-cd distribution of range cell before quadratic chirp rate correction. (e) t-cd distribution of range cell before quadratic chirp rate correction (f) RD imaging result. (g) Dechirpclean imaging result. (h) Radon-TCDS-Relax imaging result.

is about $80 \mathrm{~m} \times 80 \mathrm{~m}$. Assume MTRC phenomenon has been eliminated by the conventional. The cross range migration from third order term in phase must be considered. After translational motion compensation and keystone formatting, echo's envelope is shown as in Figure $6 \mathrm{~b}$. It can be note that the envelope has been aligned. Figure $6 \mathrm{c}$ and Figure $6 \mathrm{~d}$ show the time frequency distribution and time chirp distribution of the signal in one range cell. It can be noted that the data is of curve distribution in the time-frequency plane and of linear distribution in the time chirp plane due to the existence of the cubic phase term. Figure 6e shows the 
distribution diagram of in the time chirp plane after quadratic chirp rate estimated and compensated the cubic phase term, from which it can be noted that the data now are of linear distribution in the time -chirp plane and distributes along a beeline parallel to the time axis. The RD imaging result on the basis of Figure 6f, as we can see for the third order term in phase has not been correctly compensated only outline can be discerned and defocusing phenomenon are found, and therefore target's details cannot be distinguished. The DechirpClean imaging result is shown in Figure 6g, For the third order term are not be eliminate effectively, there are lots of fake scatterers and top tail and wing of fuselage cannot be clearly viewed. Figure $6 \mathrm{~h}$ is the Radon-TCDS-Relax imaging result, we can note that the model details is depicted fairly well in the image. For the parameter of chirp rate and its frequency have been estimated accurately. There are only few fake scatterers in the ISAR image and effectiveness of proposed algorithm has confirmed.

\section{Conclusions}

In bistatic ISAR system, the variation of bistatic angle and equivalent monostatic angel in receiver centered region has a great effect on the range envelope and cross range. The high order term in phase cannot be ignored. To achieve the high resolution target image, a new ISAR imaging method in bistatic receiver center region, referred to as Radon-TCDS-Relax, which estimating the chirp rate and a quadratic chirp rate in time chirp distribution plane is proposed in this paper. From the simulation result we can note that the image quality obtained using the proposed method outperformsthe conventional RD and RID ISAR imaging algorithms and its effectiveness of the proposed algorithm is verified too.

\section{Competing interests}

The authors declare that they have no competing interests.

\section{Acknowledgments}

This work is supported in part by the National Natural Science Foundation of China under Grants 61001204 and in part by the Science and technology Foundation of Shaanxi Province (2012JM8015) and in part by the Special Funds of the Natural Science of Shaanxi Provincial Department of Education (12JK0530,12JK0557)

Received: 31 July 2012 Accepted: 22 February 2013

Published: 16 March 2013

\section{References}

1. CC Chen, HC Andrews, Target-Motion-Induced Radar Imaging. IEEE Trans on AES 16(1), 2-14 (1980)

2. JP Marco Martorella, H John, Ambiguity Function for a Bistatic Radar. IEEE Transactions on AES 43(3), 1125-1134 (2007)

3. J Palmer, J Homer, ID Longstaff et al., ISAR imaging using an emulated multistatic radar system. IEEE Transactions on AES 41(4), 1464-1472 (2005)

4. T Tsao, M Slamani, P Varshney et al., Ambiguity Function for a Bistatic Radar. IEEE Transactions on AES 33(3), 1041-1051 (1997)

5. MI Skolnik, Radar Handbook[M] (McGraw-Hill, Inc, 1970)

6. M Xing, Z Bao, Imaging algorithm for steadily flying and maneuvering big targets. Proc. SPIE 4382, 182-190
7. GY Lu, Z Bao, Compensation of scattererser migration through resolution cell in inverse synthetic aperture radar imaging. IEE Proc Radar, Sonar Navig 147(2), 80-85(2000) (2000)

8. $Z G Z, C L Y, M D$ Xing, ISAR imaging of manoeuvring targets with the range instantaneous chirp rate technique. IET Radar Sonar Navig 3(5), 449-460 (2009)

9. $\quad Y$ Li, R Wu, M Xing, Z Bao, Inverse synthetic aperture radar imaging of ship target with complex motion. IET Radar Sonar Navig 2(6), 395-403 (2008)

10. JC O'Neill, P Flandrin, Virtues and vices of quartic time-frequency distributions. IEEE Trans. Signal Processing 48(9), 2641-2650 (2000)

11. VC CHEN, H Ling, 'Time-frequency transforms for radar imaging and signal analysis' (Artech House Inc, 2002)

12. J Li, P Stoica, Efficient mixed-spectrum estimation with applications to target feature extraction. IEEE Trans on Signal Proc 44(2), 281-29 (1996)

13. L Jian, Z Dunming, Angle and waveform estimation via RELAX[J]. IEEE Trans on AES 33(3), 1077-1086 (1997)

14. C Sun, Z Bao, Super-resolution algorithm for instantaneous ISAR imaging Electron. Lett. 36(3), 253-255 (2000)

doi:10.1186/1687-6180-2013-50

Cite this article as: Zhang et al:: High resolution ISAR imaging in receiver centered region area in bistatic radar. EURASIP Journal on Advances in Signal Processing 2013 2013:50.

\section{Submit your manuscript to a SpringerOpen ${ }^{\circ}$ journal and benefit from:}

- Convenient online submission

- Rigorous peer review

- Immediate publication on acceptance

- Open access: articles freely available online

- High visibility within the field

- Retaining the copyright to your article

Submit your next manuscript at $>$ springeropen.com 